\title{
Rails et voies des chemins de fer secondaires
}

\section{Rémy Guyot}

\section{CpenEdition}

\section{Journals}

Édition électronique

URL : https://journals.openedition.org/rhcf/2041

DOI : 10.4000/rhcf.2041

\section{Éditeur}

Rails \& histoire

Édition imprimée

Date de publication : 2 mai 2002

Pagination : 138-145

ISBN : 00996-9403

ISSN : 0996-9403

\section{Référence électronique}

Rémy Guyot, "Rails et voies des chemins de fer secondaires », Revue d'histoire des chemins de fer [En

ligne], 24-25 | 2002, mis en ligne le 13 avril 2015, consulté le 22 avril 2022. URL : http:// journals.openedition.org/rhcf/2041 ; DOI : https://doi.org/10.4000/rhcf.2041 


\section{Rémy GUYOT}

\section{Rails et voies des chemins de fer secondaires}

\section{Généralités - consistance du réseau des chemins de fer secondaires en France métropolitaine}

Les chemins de fer secondaires dénommés dans les textes officiels «chemins de fer d'intérêt local» se sont développés sur 23900 kilomètres, en moins de 50 ans entre 1870 et 1914.

Par comparaison, lors de la mise en place de la SNCF le $1^{\text {er }}$ janvier 1938, les grands réseaux totalisaient quelques 42000 kilomètres de voies.

\section{Situation en 1870}

Vers la fin du second Empire il était apparu qu'une grande partie du territoire métropolitain, située en zone rurale et en zone de montagne, ne pourrait pas être desservie rapidement par les grandes compagnies, peu désireuses de construire à grands frais des antennes « vicinales » qu'elles jugeaient obligatoirement déficitaires.

En 1870 les chemins de fer d'intérêt local ne totalisaient que 300 kilomètres, alors que les grandes compagnies dépassaient les 17000 kilomètres.

Mesures législatives destinées à permettre l'essor des chemins de fer secondaires

Le législateur, par les lois de 1865, 1880 et 1913 (voir en annexe), s'est donné les moyens de réaliser un programme ferroviaire de développement «local», laissant aux grandes compagnies la maitrise de l'extension du programme d'intérêt général.

En effet, il fut accordé aux départements, voire aux communes, la possibilité d'établir ces chemins de fer, de les exploiter ou les concéder.

Pour ménager les finances des divers partenaires - État, départements - il convenait de concevoir un réseau simple, établi suivant un programme plus modeste et plus économique que celui servant à la construction des lignes d'intérêt général exploitées par les grandes compagnies. 


\section{Réalisation du programme économique}

Pour procéder techniquement à la réalisation d'économies dans la construction de voies ferrées on peut jouer sur plusieurs paramètres :

- abaisser le rayon des courbes pour réduire le développement du tracé ; de terrains ;

- emprunter les routes ou les accotements pour limiter les achats

- diminuer les terrassements par l'adoption d'un profil en long plus important;

- mettre en place une superstructure allégée : rails de faible poids, traverses courtes, signalisation minimum, passages à niveau simplifiés, absence de clôtures...;

- adopter l'écartement de voie le plus adéquat en fonction de topographie et du trafic escompté car, plus celui-ci sera faible, plus importantes seront les économies.

Ecartements adoptés pour les chemins de fer secondaires, caractéristiques linéaires

\section{Définitions}

Trois types d'écartement furent adoptés par les constructeurs que l'on définit ainsi :

- écartement de 1,44 m désigné sous le vocable « voie normale », type grands réseaux ;

- écartement de $1,00 \mathrm{~m}$ ou « voie métrique »;

- écartement de 0,60 m ou « voie étroite ».

Les écartements de 1,44 et $1 \mathrm{~m}$ étaient prévus au cahier des charges ministériel d'août 1881 ; l'écartement de $0,60 \mathrm{~m}$ ne fut autorisé qu'en 1891.

\section{Choix d'un type d'écartement}

Le coût de construction des voies secondaires variait du simple au triple : la construction d'un kilomètre de voie unique à écartement normal revenait à 175000 francs (or), à la fin du XIX ${ }^{\mathrm{e}}$ siècle ; s'il était à écartement métrique, il coûtait 80000 francs et, à écartement étroit, 60000 francs.

Le choix de l'écartement à adopter dépendait des revenus escomptés, donc du trafic probable.

\section{Répartition par écartement des chemins de fer secondaires}

$23900 \mathrm{~km}$ sont répartis en :

- voie «normale 1,44» type grands réseaux : $2950 \mathrm{~km}(12,2 \%)$ 
- voie «métrique 1,00»: $20500 \mathrm{~km}(85,5 \%)$

- voie « étroite $0,60 »: 450 \mathrm{~km}(2 \%)$

- voies « hors normes »: $80 \mathrm{~km}(0,3 \%)$

Soit $0,75 \mathrm{~m}: 12 \mathrm{~km}$

$1,10 \mathrm{~m}: 58 \mathrm{~km}$

$0,50 \mathrm{~m}: 10 \mathrm{~km}$.

\section{Caractéristiques communes aux divers écartements}

- Les rails : leur poids est donné en kilogramme par mètre linéaire. Quant à leur type, le rail Vignole, également appelé « rail à patin », repose directement sur la traverse, tandis que le rail double champignon, également appelé « rail à coussinets », repose sur la traverse par l'intermédiaire d'un coussinet.

- Les tracés sont dit en « site propre » lorsqu'ils sont établis sur des terrains privés acquis et aménagés avec déblais, remblais et ouvrages d'art pour respecter le profil en long.

- La vitesse commerciale tient compte des temps d'arrêt et de manœuvre lors de la desserte d'une ligne.

- La distance entre arrêts situe en plan la position respective des gares, stations, ou haltes.

- Les rayons de courbes sont des rayons minimum.

- Les profils (pentes, rampes) sont des profils moyens.

\section{Chemins de fer secondaires établis en « voie normale » $(1,44 \mathrm{~m})$}

Cet écartement représente $2950 \mathrm{~km}$ de voies établis avec des rails Vignole légers (25 à $30 \mathrm{~kg}$ le mètre linéaire). Ils sont construits en site propre et $190 \mathrm{~km}$ sont électrifiés (moins de $7 \%$ ), dont $135 \mathrm{~km}$ en courant continu et $55 \mathrm{~km}$ en courant alternatif.

La vitesse commerciale atteinte sur ces voies est de $25 \mathrm{~km} / \mathrm{h}$, la distance moyenne entre les arrêts de $3,5 \mathrm{~km}$.

Ils sont tracés avec des courbes de rayon supérieures à $200 \mathrm{~m}$, sauf aux abords des gares; et les profils sont de l'ordre de $20 \mathrm{~mm}$ par mètre linéaire.

Ces voies ne se développèrent que dans les régions à gros trafic (principalement minier) comme le Nord-Pas-de-Calais ou dans les régions à topographie facile - Gironde, Landes...

Il en fut construit également aux abords de villes importantes où ils jouaient le rôle de tramway, dans les départements de Seine-et-Oise, Rhône, Bouches-du-Rhône, Isère. 


\section{Chemins de fer secondaires établis en « voie métrique » $(1,00 \mathrm{~m})$}

La voie métrique $(1,00 \mathrm{~m})$ compte $20500 \mathrm{~km}$.

$19000 \mathrm{~km}$ sont armés de rails Vignole légers $(15 \mathrm{à} 25 \mathrm{~kg}$ le mètre linéaire), soit $95 \%, 1000 \mathrm{~km}$ avec rails double champignon sur coussinets d'un poids de 23 à $30 \mathrm{~kg}$ (Corse, Val-de-Loire, Bretagne). En chaussée, des rails à profils spéciaux incluent l'ornière (Broca). Ils se répartissent presque également entre lignes en site propre : $10500 \mathrm{~km}$ et lignes sur chaussée ou accotement : $10000 \mathrm{~km}$.

$2150 \mathrm{~km}$, soit $10 \%$, sont électrifiés, $1600 \mathrm{~km}$ en courant continu, $550 \mathrm{~km}$ en courant alternatif.

La vitesse commerciale n'atteint que $20 \mathrm{~km} / \mathrm{h}$, la distance moyenne entre les arrêts est de $3,5 \mathrm{~km}$ (10 km pour la Corse). Le rayon des courbes baisse à environ $50 \mathrm{~m}$ et les profils sont de l'ordre de $30 \mathrm{~mm}$ par $\mathrm{ml}$, atteignant jusqu'à $80 \mathrm{~mm}$ (sans crémaillère).

Avec un kilométrage important, la voie métrique devint la « vedette » des chemins de fer secondaires français car son coût était acceptable pour les finances départementales et son établissement technique découlant du programme économique décrit ci-dessus permettait à ce type de voie de pénétrer au cour des campagnes, de desservir au plus près les localités rurales selon le désir de leurs habitants.

Toutefois, ce choix imposait deux restrictions : la première était l'impossibilité d'aller vite à cause des courbes prononcées; mais une vitesse de 15 à $25 \mathrm{~km}$ à l'heure paraissait très suffisante, surtout à la traversée des bourgs et villages, et se conciliait avec les rayons de courbure de la voie.

La seconde était la nécessité du transbordement des marchandises aux jonctions avec les grands réseaux établis en voie normale, mais pour un trafic jugé faible ; l'inconvénient paraissait minime à l'époque.

La répartition de la voie métrique sur le territoire métropolitain fut inégale : les régions les mieux pourvues sont la Bretagne, les Charentes, le Val-de-Loire, Rhône-Alpes où le kilométrage par département dépasse $400 \mathrm{~km}$; les régions les moins bien dotées sont l'Auvergne, les Cévennes avec moins de 100 kilomètres par département.

Pour modeste qu'il fut, le mode d'électrification de certaines lignes (de montagne principalement) se montra d'avant-garde par rapport à la technique électrique bien peu développée des grandes compagnies.

Il permit dans les Alpes et dans les Pyrénées de circuler sur des rampes de près de 80 millimètres par mètre. 
Il donna l'occasion au département de la Haute-Vienne d'être novateur par l'utilisation du courant monophasé à la fréquence industrielle de $25 \mathrm{~Hz} / \mathrm{s}$ sur l'ensemble de son réseau (340 kilomètres).

\section{Chemins de fer secondaires établis en « voie étroite » $(0,60 \mathrm{~m})$}

La voie étroite de $0,60 \mathrm{~m}$ compte $450 \mathrm{~km}$ établis avec des rails Vignole de 9,5 kg sur traverses métalliques (système Decauville) ou de $15 \mathrm{~kg}$ sur traverses bois.

Elle est en site propre sur $45 \mathrm{~km}$ (Loiret, $10 \%$ ) et en chaussée ou accotement sur $400 \mathrm{~km}(90 \%)$. La vitesse commerciale baisse à $15 \mathrm{~km} / \mathrm{h}$ $(10 \mathrm{~km} / \mathrm{h}$ sur les voies militaires), la distance entre les arrêts à $2,7 \mathrm{~km}$.

Les courbes ont sur ces voies entre 30 et $40 \mathrm{~m}$ de rayon et les profils sont de 25 à $40 \mathrm{~mm}$ par mètre linéaire.

Avec seulement 450 kilomètres, les réalisations en voie de $0,60 \mathrm{~m}$ sont restées modestes : l'agrément tardif donné par les autorités à leur construction, dix ans après le cahier des charges de 1881, semble en être la cause.

Ces réalisations concernent :

- les trains de plage établis en bord de mer dans les départements de la Charente-maritime (abords de Royan), du Morbihan (Etel-LaTrinité), de la Somme (For-Mahon), du Pas-de-Calais (Paris-Plage) ;

- des lignes isolées établies dans les départements du Tarn $(50 \mathrm{~km})$, du Loiret (45 km), de Savoie (30 km pour l'Étoile de Chambéry);

- un réseau cohérent et étoffé, celui du Calvados $(221 \mathrm{~km})$ construit de 1892 à 1906, qui dessert toute la côte balnéaire et la relie également aux villes de Caen, Falaise, Bayeux ;

- Le réseau de guerre utilisé après 1918 par le ministère des Régions libérées (M.R.L.).

Bien que n'apparaissant pas dans les statistiques, les anciens réseaux de voies de 0,60 utilisés par les belligérants furent récupérés et installés pour rétablir dans les régions sinistrées les conditions du retour à une vie normale. En effet, dans les zones du front, du Nord aux Vosges, la quasi-totalité des transports routiers et ferroviaires classiques était paralysée : il s'avéra que le meilleur moyen de démarrer la reconstruction était d'utiliser les nombreuses voies étroites abandonnées sur les champs de bataille.

Le réseau fut remis en service, complété par des raccordements, des stations de transbordement avec les grands réseaux, ainsi qu'avec les voies navigables. Des embranchements particuliers permettaient l'accès aux carrières, sablières, cimenteries, briqueteries, fours à chaux... La plupart de ces lignes avait pour vocation le transport des marchandises ; 
toutefois, certaines admettaient un service voyageurs, souvent minimum, réduit à deux ou trois jours par semaine, voire à un seul passage hebdomadaire. A partir de 1923 ces lignes furent soit déferrées, soit cédées aux départements. Elles disparurent pratiquement vers 19281930.

Évolution du développement des chemins de fer secondaires déclin - état actuel

\section{Évolution}

La figure 1 résume l'évolution de l'ensemble des chemins de fer secondaires de 1870 à nos jours.

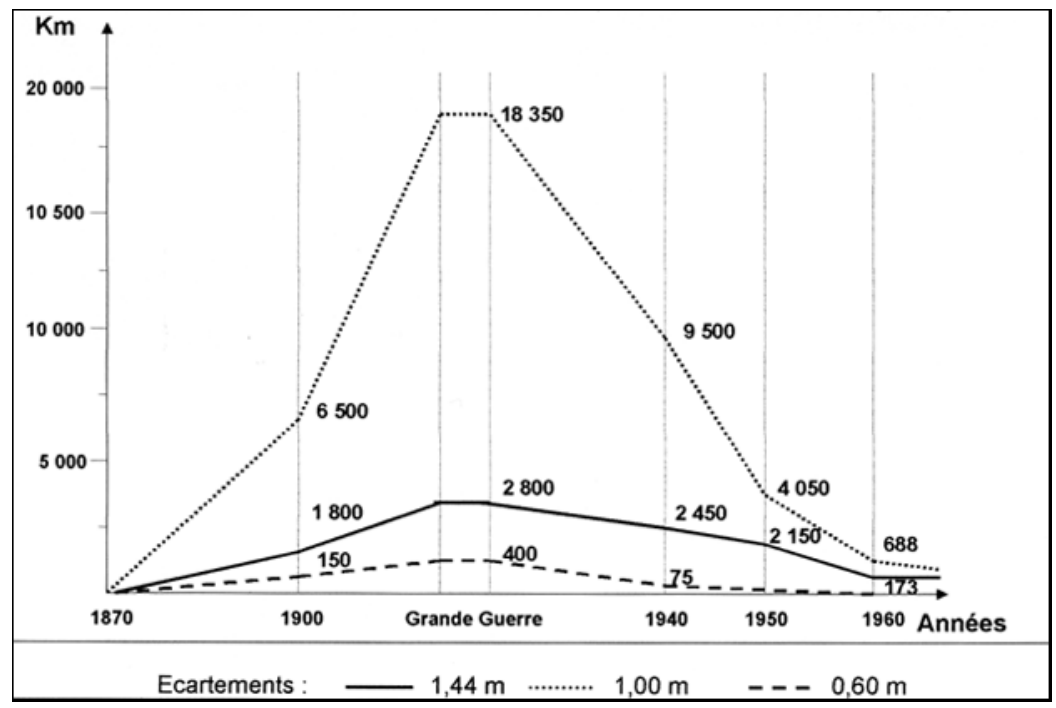

Figure 1 : Chemins de fer secondaires. Évolution des longueurs en service (en km de 1870 à nos jours).

Leur construction fut lente à démarrer sous le régime de la loi de 1865. Elle ne débuta vraiment qu'avec la mise en application de la loi de 1880 pour atteindre son apogée en 1914. La Grande Guerre stoppa tous les travaux de développement et bien des petites lignes de l'arrière du front furent réquisitionnées, déferrées et privées de leurs engins de traction pour les besoins de l'armée. 


\section{Déclin}

Le déclin commença dès la fin du conflit et ce, malgré la construction entre 1918 et 1940 de 1900 kilomètres de nouvelles voies. Le déclin fut aussi rapide que l'expansion.

L'augmentation du prix des combustibles, des salaires, l'abaissement du temps de travail furent fatals à de nombreuses compagnies malgré un relèvement important des tarifs des transports (atteignant parfois $500 \%$ ). Certains départements reprirent les concessions abandonnées par leurs exploitants mais ce fut peine perdue, le déficit continuait et l'arrivée en force de l'autocar moins coûteux et plus rapide accéléra les fermetures et les déposes. C'est ainsi que l'on est passé, de 1918 à 1960 :

- pour la voie normale de 2800 à 200 kilomètres ;

- pour la voie métrique de 18350 à 700 kilomètres ;

- pour la voie étroite de 400 à 40 kilomètres.

\section{État actuel}

Que reste-t-il au début de ce XXI ${ }^{\mathrm{e}}$ siècle de l'ensemble des chemins de fer secondaires?

En voie normale environ 200 kilomètres de desserte marchandises ou en embranchements particuliers, soit $8 \%$ de l'ensemble construit.

En voie métrique environ 700 kilomètres, dont des lignes voyageurs exploitées soit par la SNCF, soit par d'autres entreprises ferroviaires dont :

- Salbris-Romorantin-Luçay-le-Mâle : 67 km

- chemins de fer corses : $231 \mathrm{~km}$

- chemin de fer de Provence : $150 \mathrm{~km}$

- chemin de fer de Cerdagne : $62 \mathrm{~km}$

- Saint-Gervais-Vallorcine : $51 \mathrm{~km}$

- réseau breton (en partie) : $90 \mathrm{~km}$.

Le reste en « petits trains touristiques »: Vivarais, La Mure, Baie de Somme... : soit 3\% de l'ensemble construit.

En voie étroite seuls subsistent quelques trains touristiques de faible parcours (2 à 7 kilomètres) pour une longueur totale d'environ 40 kilomètres, soit moins de $10 \%$ de l'ensemble construit.

\section{Conclusion}

La construction des lignes d'intérêt local fut-elle en son temps la meilleure solution technique à la desserte du monde rural au début du $\mathrm{XX}^{\mathrm{e}}$ siècle? On peut répondre oui. 
En effet, à cette époque, seul le pas du cheval rythmait la vie des campagnes et le développement routier était inexistant (il a fallu attendre les années 1920 pour voir l'essor du camionnage). Ainsi, pendant 30 ans, le mode ferroviaire d'intérêt local a permis la mise en valeur des zones rurales à faible population, mais bien irriguées par les lignes secondaires, les faisant ainsi participer et bénéficier à l'essor économique de notre pays. dre : non.

Ce type de chemin de fer pouvait-il perdurer? On peut répon-

En effet, tracées le long des routes passant au milieu des villages n'admettant qu'une vitesse archaïque, cohabitant dans un même espace, de plus en plus difficilement, avec les autres modes de transport, beaucoup de ces lignes locales n'auraient pu survivre même avec des moyens financiers accrus.

Seules ont subsisté les lignes établies en site propre susceptibles d'être modernisées et rendues adaptables aux besoins des voyageurs de notre $\mathrm{XXI}^{\mathrm{e}}$ siècle.

Faut-il en regretter la disparition ? La raison dit : non et le cœur dit : souvenez-vous!

Évoquer leur existence passée n'est-ce pas, en plus du souvenir, faire aussi preuve de reconnaissance? 